\title{
Treinamento em primeiros socorros com alunos do ensino regular: relato de experiência
}

\author{
First aid training with regular school students: experience report \\ Capacitación em primeros auxílios con estudiantes de escuelas regulares: informe de experiencia
}

Recebido: 29/10/2021 | Revisado: 04/11/2021 | Aceito: 09/11/2021 | Publicado: 14/11/2021

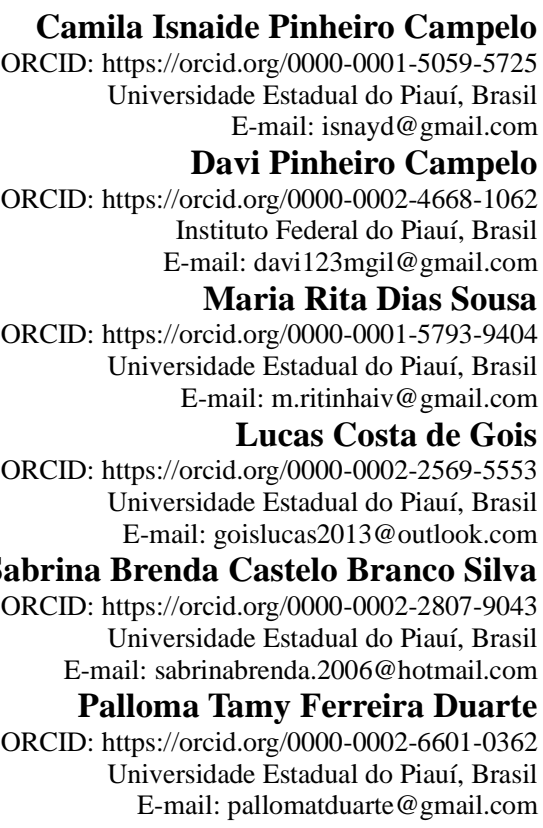

\section{Resumo}

Objetivo: apresentar um relato sobre a experiência vivida por graduandos em enfermagem ante a educação em saúde sobre primeiros socorros para alunos de ensino regular. Metodologia: Estudo descritivo, fundamentado em um relato das experiências vivenciadas por graduandos em enfermagem durante a execução de um projeto de iniciação científica da Universidade Estadual do Piaú. Desenvolvido em uma escola de tempo integral, no período de agosto a outubro de 2019, com amostra de 34 voluntários. Consistiu-se na avaliação da eficácia do treinamento, por meio aplicação de pré e pós-teste, seguido de ações educativas com aulas expositivas e dialogado, sucedidas de momentos práticos, onde os participantes eram colocados em situações hipotéticas e precisavam intervir. Resultados e discussão: Notou-se o potencial de disseminação de informações que os alunos possuíam facilitando, desta forma, a propagação dos cuidados iniciais prestados a uma vítima de acidente ou mal súbito. Conclusão: Afirma-se que os aprendizados e experiências alcançadas foram inestimáveis para os autores da pesquisa.

Palavras-chave: Primeiros socorros; Educação em saúde; Emergências; Educação continuada em Enfermagem.

\begin{abstract}
Objective: to present a report on the experience of nursing undergraduates in health education on first aid for regular education students. Methodology: Descriptive study based on an account of the experiences lived by undergraduate nursing students during the execution of a scientific initiation project at the State University of Piauí. Developed in a full-time school, from August to October 2019 with a sample of 34 volunteers. It consisted of evaluating the effectiveness of the training through the application of pre- and post-tests followed by educational actions with lectures and dialogue, followed by practical moments of practical moments, where participants were placed in hypothetical situations and needed to intervene. Results and discussion: It was noted the potential for disseminating information that students had, thus facilitating the spread of initial care provided to a victim of accident or sudden illness. Conclusion: It is stated that the learning and experiences achieved were invaluable for the authors of the research.
\end{abstract}

Keywords: First aid; Health education; Emergencies; Education Nursing continuing. 


\begin{abstract}
Resumen
Objetivo: presentar un informe sobre la experiencia de los estudiantes de enfermería en educación para la salud en primeros auxilios para estudiantes de educación regular. Metodología: Estudio descriptivo, basado en un relato de las experiencias vividas por estudiantes de pregrado de enfermería durante la ejecución de un proyecto de iniciación científica en la Universidad Estatal de Piauí. Desarrollado en una escuela de tiempo completo de agosto a octubre de 2019 con una muestra de 34 voluntarios. Consistió en evaluar la efectividad de la formación, mediante la aplicación de pre y post-pruebas, seguidas de acciones educativas con charlas y diálogo, seguidas de momentos prácticos, donde los participantes fueron colocados en situaciones hipotéticas y necesitaban intervenir. Resultados y discusión: Se notó el potencial de difusión de información que tenían los estudiantes facilitando así la difusión de la atención inicial brindada a una víctima de accidente o enfermedad repentina. Conclusión: Se afirma que los aprendizajes y experiencias logradas fueron invaluables para los autores de la investigación.
\end{abstract}

Palabras clave: Primeros auxilios; Educación en salud; Emergencias; Educación continua en Enfermería.

\title{
1. Introdução
}

Os primeiros socorros são medidas iniciais diante de uma situação de emergência, sejam acidentes ou males súbitos, aplicadas no local ou próximo, objetivando a manutenção dos sinais vitais e evitando agravamentos das lesões, aliviando o sofrimento, proporcionando melhores condições à vítima até que a assistência especializada esteja disponível (Ferreira, Alves, Souto, Virgínio, Silva Júnior \& dos Santos, 2017).

Considera-se que este socorro inicial pode ser desenvolvido por qualquer pessoa que esteja treinada e próxima ao acidentado, desde que seja capaz de executar esta assistência. Embora simples, os cuidados necessitam de precisão e habilidade, haja vista que uma má execução dos primeiros socorros é tão prejudicial quanto a falta deles (Aranha, Barsotti, Silva, Oliveira \& Pereira, 2019; Calandrim et al., 2017).

Portanto, o socorro prestado nas primeiras horas após um acidente é de suma importância, pois é um período crítico, sendo possível garantir a recuperação de sequelas e danos ou a sobrevivência das pessoas feridas. Entretanto, a realidade retrata uma população com déficit de informação quanto as noções dos primeiros socorros, impossibilitada de agir adequadamente (Dantas et al., 2018; Silva, Tassara, Ansaloni, Moraes, Oliveira, \& Matias, 2020).

Assim, ressalta-se que um pré-atendimento adequado, prestado por pessoas leigas, mas treinadas, contribui para evitar sequelas e/ou que o indivíduo evolua para o óbito. Contudo, essa ausência de procedimentos imediatos, somada a inadequação destes, quando realizados, são as principais causas de óbitos externos ao ambiente hospitalar, partindo do pressuposto que cada acidente necessita de uma intervenção específica, para isso a educação continuada ou permanente faz-se necessário (Cardoso, Soares, Calixto, Carvalho, Durante \& Veloso, 2017; Galindo Neto, Caetano, Barros, Silva \& Vasconcelos, 2017).

Neste ínterim, a violência e os acidentes são interpretados como um objeto de abordagem intersetorial, e a saúde, por sua vez, tende a incorporar esses conceitos em seu amplo campo de atuação. Os dados da literatura revelam que as políticas de promoção da saúde devem, de maneira urgente, priorizar uma educação sólida (Guimarães, 2011; Silva, Costa, Furtado, Tavares \& Costa, 2017).

Diante deste contexto, a escola há muito deixou de ter um papel meramente acadêmico e desde então contribui com a formação do ser como um todo, parte de uma sociedade. Destarte, compreende-se que o ambiente escolar é favorável para a educação em saúde, objetivando a minimização dos agravamentos, decorrentes de acidentes e males súbitos (Dantas et 1., 2018; Silva, Costa, Furtado, Tavares \& Costa, 2017).

Ressalta-se ainda que, o ensino de primeiros socorros necessita ser amplamente ensinado, de forma a democratizá-lo, sendo mais abordado com a população, passando a ser parte do conteúdo curricular de todos os alunos, haja vista que aprender medidas básicas, para prestar um socorro imediato, ajudaria os indivíduos a atuar com maior segurança e competência diante de situação de emergência (Silva, Costa, Furtado, Tavares \& Costa, 2017; Silva, Nunes, Moreira \& Costa, 2018).

Desta forma, a educação e o potencial de propagação das informações, que estes alunos possuem, torna-se fundamental envolvê-los em soluções de casos emergenciais, para que a disseminação de conhecimento científico se faça 
possível. Por isso, justifica-se uma metodologia que possa transmitir, aos alunos de escolas do ensino integral, as primeiras condutas de atendimento, que antecedem a chegada do socorro às vítimas (Silva, Nunes, Moreira \& Costa, 2018).

O objetivo deste artigo foi apresentar um relato sobre a experiência vivida por graduandos em enfermagem ante a educação em saúde sobre primeiros socorros para alunos de ensino regular.

\section{Metodologia}

Trata-se de um estudo descritivo, fundamentado em um relato das experiências vivenciadas por graduandos em enfermagem durante a execução de um projeto de iniciação científica da Universidade Estadual do Piauí.

O público alvo foi composto por alunos do ensino regular em uma escola pública de Teresina, Piauí. A escola em questão funcionava em regime integral, então o acesso aos alunos foi organizado junto à coordenação que disponibilizou espaço físico e horário específico uma vez por semana.

A pesquisa consistiu na avaliação da eficácia do treinamento por meio de aplicação de questionário de caráter objetivo, contendo 20 questões, no primeiro encontro, seguido de ações educativas, em encontros posteriores, divididos em um cronograma e apresentados em um período de 20-30 minutos. Os momentos seguintes à exposição do material didático, também com a mesma duração, eram destinados as atividades práticas e elucidação das dúvidas.

Foram abordados conteúdos sobre noções básicas de primeiros socorros, como noções de biossegurança, acionamento correto do serviço de atendimento móvel de urgência, manobras durantes situações de engasgo e parada cardiorrespiratória, crise convulsiva, bem como ações diante de situações envolvendo choque elétrico, intoxicação exógena, queimaduras, hemorragias e fraturas. As aulas eram expositivas e dialogadas, sucedidas de momentos práticos, onde os participantes eram colocados em situações hipotéticas e precisavam intervir. Por fim, aplicou-se o questionário novamente, constituindo o pósteste.

As atividades foram desenvolvidas no período de agosto a outubro de 2019, por um grupo de cinco graduandos em enfermagem e a professora orientadora, nas dependências da escola. A população do estudo foi composta por 34 alunos da $1^{a} \mathrm{e}$ $2^{\text {a }}$ série do ensino médio, da referida escola. A amostragem se deu por conveniência, já que as aulas dependiam da disponibilidade dos participantes.

É importante mencionar que neste artigo não foram inclusos dados dos participantes deste projeto.

\section{Resultados e Discussão}

Após a aplicação do pré-teste, constituído por 20 questões objetivas, foi apresentado o cronograma de datas e horários previamente disponibilizados pela instituição e apresentado os nove temas, descritos no quando 1 logo abaixo, que seriam expostos no decorrer dos dias

Quadro 1: Assuntos ministrados no treinamento sobre noções de primeiros socorros.

\begin{tabular}{|l|}
\hline Noções e princípios básicos de biossegurança \\
\hline Apresentação do Serviço de Atendimento Móvel de Urgência (SAMU) \\
\hline Engasgamento em lactentes e adultos \\
\hline Parada cardiorrespiratória em lactentes e adultos (Suporte básico de vida para leigos) \\
\hline Fraturas, luxações, entorses e imobilizações osteomusculares \\
\hline Queimaduras e choque elétrico \\
\hline Situações de hemorragias \\
\hline Crise convulsiva \\
\hline Intoxicação exógena \\
\hline
\end{tabular}

Fonte: Autores (2021). 
Os conteúdos abordados foram explanados na sequência já descrita no quadro supracitado com metodologia ativa, de forma a permitir a total integração dos alunos. Para tal, foram utilizados recursos audiovisuais para projeção do material didático. Antes da apresentação deste material, os participantes eram encorajados a expressar seus conhecimentos prévios, de forma a estabelecer o nível de entendimento e diagnosticar os déficits e dúvidas mais evidentes. Nesses momentos eles não apenas pontuavam suas dúvidas e conhecimentos, mas relatavam eventos vivenciados ou que tomaram ciências, mas sempre sobre pessoas próximas, correlacionados com os temas e externavam suas experiências e tornou-se rotina durante o desenvolvimento da pesquisa, enriquecendo as discussões de modo inimaginável e tornando a troca de saberes bidirecional.

Em seguida os graduandos de enfermagem envolvidos, em conjunto com a professora orientadora, abordavam o tema, buscando sempre a interação com os voluntários, permitindo uma troca de saberes. Em um segundo momento, encenava-se uma situação dentro da temática da aula e a turma era convidada a resolver o incidente ou apontar soluções para um desfecho correto diante de situações de urgência e emergência. E no último encontro, o questionário foi reaplicado com os mesmos critérios utilizados no pré-teste, em seguida os voluntários da pesquisa receberam a oportunidade para um feedback direcionado aos pesquisadores, onde apresentaram suas opiniões e sugestões de melhoria na metodologia.

Durante este momento de reflexão, fez-se perceptível como, por vezes, os alunos de cursos da saúde se veem tão familiarizados com as temáticas que envolvem primeiros socorros que chegam a superestimar o conhecimento da população, em contrapartida, esta, por vezes, nunca teve um contato sequer com um treinamento básico, então isso acaba impactando e sensibilizando estes futuros profissionais, tornando-os mais atentos a sociedade e suas deficiências e fomentando interesse por educação em saúde.

Visto isso, compreende-se que a saúde e a educação são um binômio inseparável e de certa forma, subalterno, visto que para se alcançar educação, necessita-se da saúde, e a saúde só é possível quando se tem satisfatória educação. Considera-se que ambas possuem natureza político-social, englobando os direitos e deveres das populações, em diferentes contextos, como um todo e são compreendidas como mendicâncias sociais e, juntamente a comunidade, tem o direito de usufruir e o dever de corroborar para a concretização dos direitos, garantidos pelas instituições governamentais (Lima, Moreira, Martins \& Costa, 2021).

Ademais, a promoção de educação voltada para primeiros socorros, objetivando treinar pessoas leigas, garante o empoderamento da população, facilitando a tomada de decisões e ainda encurta a comunicação desta pessoa com um profissional de saúde, que conseguirá lhe passar informações e delegar funções que auxiliem no atendimento prévio (Ferreira, Alves, Souto, Virgínio, Silva Júnior \& dos Santos, 2017; Santos, Santos, Macedo, Freitas \& Freitas, 2021).

Grimaldi et al. (2020) desenvolveram um estudo e avaliaram por meio de pré e pós-testes o treinamento em duas escolas, publica e uma privada, sobre noções básicas de primeiros socorros. No pós-teste a média do percentual de acertos das questões na escola pública foi de 70,0\% e na escola particular foi de $85,5 \%$, valores superiores aos encontrados no pré-teste. Demonstrando que em ambas as escolas os estudantes foram capazes de agregar a maior parte do conteúdo discutido durante as oficinas.

Neste contexto, a escola mostra-se como um ambiente propício para o aprendizado sobre primeiros socorros, visto que é um dos principais locais onde as relações interpessoais ocorrem cotidianamente e público alvo está em idade propícia ao aprendizado (Chehuen Neto et al., 2016; Oliveira, Leão Júnior \& Borges, 2015). Cita-se ainda, que foi notório o potencial de disseminação de informações que estes alunos possuíam, facilitando, desta forma, a propagação dos cuidados iniciais prestados a uma vítima de acidente ou mal súbito.

Ressalta-se que os países desenvolvidos já incluíram em suas grades curriculares a disciplina de primeiros socorros, tendo como objetivo aumentar a conscientização e interesse sobre as situações de emergência, possibilitando treinamento correto a toda população, desde a infância. Esta capacitação deve levar em consideração a realidade em que a população está 
inserida, para escolher temas mais apropriados para serem abordados com ênfase (Crippa \& Silva, 2020; Matos, Souza \& Alves, 2016).

Portanto, durante o planejamento das aulas a serem apresentadas aos voluntários da pesquisa, os autores recorreram à literatura local para estabelecer os temas mais necessários e a abordagem mais apropriada para cada um, visando suprir os défices identificados e estabelecer uma comunicação simples e acessível aos participantes.

Ademais, o Programa Saúde na Escola (PSE) é pontuado como uma estratégia utilizada para que haja uma integração constante entre as políticas e ações de educação e de saúde, com a participação da comunidade escolar e as equipes de saúde (Cabral \& Oliveira, 2019; Lima et al., 2020; Lopes, Nogueira \& Rocha, 2018). Por consequência, o conhecimento adquirido proporciona efetiva ação diante de situações que exijam intervenções e prevenção dos agravamentos à situação de saúde da vítima. Á vista disso, propõe-se o aprimoramento e a efetivação de políticas públicas que proporcionem a sociedade noções básicas de primeiros socorros.

\section{Conclusão}

Em síntese, nota-se a constante necessidade de educação em saúde, principalmente no contexto de situações de urgência e emergência, para a população em geral, utilizando-se das estratégias de ensino que são plenamente adaptáveis, dependendo da particularidade do público alvo da ação. E que a ambiência escolar potencializa o aprendizado e a disseminação deste conhecimento, visto a rede de contatos que os envolvidos possuem. Além de ser um ambiente onde ocorrem situações que exigem conhecimentos básicos de primeiros socorros.

Afirma-se que os aprendizados e experiências alcançadas foram inestimáveis para os autores da pesquisa, uma vez que exerceram influência sobre a realidade social desta comunidade, contribuindo para a prevenção de agravos de saúde e a estes foi possível a visualização do processo de ensino teórico-prático durante a produção e ministração das aulas e ainda mais nos momentos de diálogo com os voluntários, promovendo também resultados que geram embasamento científico pra a manutenção de estudos com este perfil.

Destaca-se ainda, a importância de pesquisas nesta área, não apenas na busca por novos dados, mas visando traduzir o que já é sabido, otimizando a prestação do cuidado dispensado ao indivíduo.

\section{Referências}

Aranha, A. L. B., Barsotti, G. M., Silva, M. P., Oliveira, N. M. \& Pereira, T. Q. (2019). Revisão integrativa: importância da orientação de técnicas de primeiros socorros para leigos. Revista Científica Multidisciplinar Núcleo do Conhecimento, 4(6),218-242.

Cabral, E. V. \& Oliveira, M. D. F. A. (2019). Primeiros socorros na escola: conhecimento dos professores. Revista Práxis, 11(22).

Calandrim, L. F.; Santos, A. B., Oliveira, L. R.; Massaro, L. G.; Vedovato, C. A.; Boaventura, A. P. (2017). Primeiros socorros na escola: treinamento de professores e funcionários Revista da Rede de Enfermagem do Nordeste. Revista Universidade Federal do Ceará, 18 (3), $292-299$. https://doi.org/10.15253/2175-6783.2017000300002.

Cardoso, R. R., Soares, L. G. B., Calixto, F. R. P., Carvalho, L. F. S., Durante, R. V.\& Veloso, R. C. (2017). Suporte básico de vida para leigos: uma revisão integrativa. Revista Unimontes Científica, 19(2).

Chehuen Neto, J. A., Brum, I. V., Pereira, D. R., Santos, L. G., Moraes, S. L. \& Ferreira, R. E. (2016). Conhecimento e Interesse sobre Suporte Básico de Vida entre Leigos. International Journal of Cardiovascular Sciences, 29(6), 443-2. 10.5935/2359-4802.20160064.

Crippa, 1. G \& Silva, 1. I. A. (2020). O conhecimento dos professores de educação física em primeiros socorros em um município do Alto Tietê - SP. Revista Científica da FAMINAS, 15(1), 22-38.

Dantas, R. A. N., Dantas, D. V., Silva, I. R. N., Araújo,N. M., Laurentino, A. M. A., Nunes, H.M. A. \& Ribeiro, M. C. O. (2018). Abordagem dos primeiros socorros na escola: crianças, adolescentes e professores aprendendo a salvar vidas. Enfermagem Brasil, $17(3), 259$.

Ferreira, M., Alves, S., de Souto, C. G., Virgínio, N. de, Silva Júnior, J. N. de, \& dos Santos, A. (2017). O leigo em primeiros socorros uma revisão integrativa. Revista De Ciências Da Saúde Nova Esperança, 15(3), 12-20. https://doi.org/10.17695/revcsnevol15n3p12-20. 
Research, Society and Development, v. 10, n. 14, e592101422492, 2021

(CC BY 4.0) | ISSN 2525-3409 | DOI: http://dx.doi.org/10.33448/rsd-v10i14.22492

Galindo Neto, N. M., Caetano, J. A., Barros, L. M., Silva, T. M. \& Vasconcelos, E. M. R. (2017). Primeiros socorros na escola: construção e validação de cartilha educativa para professores. Acta Pauista del Enfermagem 30(1), 87-93. https://doi.org/10.1590/1982-0194201700013.

Grimaldi, M. R. M, Gonçalves, L. M. S, Melo, A. C. O. S., Melo, F. I., Aguiar, A. C. S. C. \& Lima, M. M. N. (2020). A escola como espaço para aprendizado sobre primeiros socorros. Revista de Enfermagem UFSM - REUFSM, 10(20), 1-15. 10.5902/2179769236176.

Guimarães, F. V. N. Educação em saúde: capacitação em primeiros socorros no ambiente escolar. 2011. Monografia (especialização). Universidade Federal de Minas Gerais. 32f.

Lima, M. G. Q., Oliveira, L. X., Morais, A. E., Pinto, B. N. S. L., Hollanda, L. E., Lidório Júnior \& R. A. (2020). Disseminação de informações sobre primeiros socorros e prevenção de acidentes em uma comunidade ribeirinha. Revista Brasileira em Promoção Saúde, 33. 10.5020/18061230.2020.10053.

Lima, P., Oliveira, T., Moreira, A., Moreira, R., Martins, E., \& Costa, A. (2021). Primeiros socorros como objeto de educação em saúde para profissionais de escolas municipais. Revista de Enfermagem da UFSM, 11 (10). https://doi.org/10.5902/2179769243292.

Lopes, I. E, Nogueira, J. A. D. \& Rocha, D. G. (2018). Eixos de ação do Programa Saúde na Escola e Promoção da Saúde: revisão integrativa. Saúde debate,42(118), 773-789. 10.1590/0103-1104201811819.

Matos, D. O. N., Souza, R. S. \& Alves, S. M. (2016). Inclusão da disciplina de primeiros socorros para alunos do ensino básico. Revista Interdisciplinar, 9(3), 68-178.

Oliveira, R. A., Leão Junior, R. \& Borges, C. C. (2015). Situações de primeiros socorros em aulas de educação física em municípios do sudoeste de goiás. Enciclopédia biosfera. 11(20), $72-77$.

Santos, N. S., Santos, G. A., Macedo, L. F. M. S., Freitas, J. C. \& Freitas, A. C. (2021). Percepção de alunos do ensino médio sobre primeiros socorros. Research, Society and Development, 10(7). http://dx.doi.org/10.33448/rsd-v10i7.15465.

Silva, B. K. M., Tassara, K. R., Ansaloni, L. V. S., Moraes, P. H. A., Oliveira, R. A. \& Matias, P. R. S. (2020). O conhecimento acerca do suporte básico de vida: uma revisão integrativa. Brazilian Journal of Development, 6(9), 72021-72039. https://doi.org/10.34117/bjdv6n9-593.

Silva, D. P, Nunes, J. B. B., Moreira, R. T. F. \& Costa, L. C. (2018). Primeiros socorros: objeto de educação em saúde para professores. Revista de enfermagem UFPE, 12(5), 1444-53.

Silva, L. G. S., Costa, J. B., Furtado, L. G. S., Tavares, J. B. \& Costa, J. L. D. (2017). Primeiros socorros e prevenção de acidentes no ambiente escolar: intervenção em unidade de ensino. Enfermagem em Foco [Internet], 8 (3), 25-29. https://doi.org/10.21675/2357-707X.2017.v8.n3.893. 